\title{
Performance modelling based on value analysis for improving product development process architecture
}

\author{
Yin, F.P. ${ }^{a, b}$, Gao, Q. ${ }^{a, b,{ }^{*}}$, Ji, X. ${ }^{a, b}$ \\ ${ }^{a}$ School of Mechanical Engineering, Shandong University, Jinan, China \\ ${ }^{b}$ Key Laboratory of High Efficiency and Clean Mechanical Manufacture, Shandong University, Ministry of Education, Jinan, \\ China
}

\begin{abstract}
A B S T R A C T
Improving the architecture of product development process (PDP) is an effective approach to improve PDP performance. However, performance is difficult to model because the criterion of performance such as development cost, time and product quality are usually contradictory. The objective of this paper is to use process value as the evaluation indicator of PDP performance. The process value of PDP, as well as the ratio of process function and process cost, is discussed and its quantitative method is proposed. The process function is defined as the process effectiveness which considers the importance of each activity of PDP, and its evaluation methods based on rework theory and quality function deployment (QFD) are given. The simulation method is used to illustrate the proposed model and analyze the relation between architecture and process value of PDP, and an optimization model for PDP architecture is provided. With the model, we can get a suitable PDP architecture to balance the cost and product function during product development.
\end{abstract}

(C) 2017 PEI, University of Maribor. All rights reserved.

\begin{tabular}{l} 
A R T I C L E I N F O \\
\hline Keywords: \\
Product development process \\
Process performance \\
Process architecture \\
Value analysis \\
Effectiveness \\
Modelling \\
*Corresponding author: \\
gaoqi@sdu.edu.cn \\
(Gao, Q.) \\
Article history: \\
Received 20 October 2016 \\
Revised 15 February 2017 \\
Accepted 18 February 2017
\end{tabular}

\section{References}

[1] Unger, D., Eppinger, S. (2011). Improving product development process design: A method for managing information flows, risks, and iterations, Journal of Engineering Design, Vol. 22, No. 10, 689-699, doi: 10.1080/ $\underline{09544828.2010 .524886 .}$.

[2] Fu, L., Jiang, P., Cao, W. (2013). Modeling and performance analysis of product development process network, Journal of Network and Computer Applications, Vol. 36, No. 6, 1489-1502, doi: 10.1016/i.jnca.2013.02.006.

[3] Browning, T.R., Eppinger, S.D. (2002). Modeling impacts of process architecture on cost and schedule risk in product development, IEEE Transactions on Engineering Management, Vol. 49, No. 4, 428-442, doi: 10.1109/ tem.2002.806709.

[4] von Hippel, E. (1990). Task partitioning: An innovation process variable, Research Policy, Vol. 19, No. 5, 407-418, doi: 10.1016/0048-7333(90)90049-c.

[5] Cedergren, S., Wall, A., Norström, C. (2010). Evaluation of performance in a product development context, Business Horizons, Vol. 53, No. 4, 359-369, doi: 10.1016/j.bushor.2010.03.001.

[6] Huang, H.-C., Fu, T.-T. (2006). On the quantitative evaluation and simulation of product development processes, In: The 36 th CIE Conference on Computers \& Industrial Engineering, Kaohsiung City, Taiwan, 532-543.

[7] Pun, K.F., Chin, K.S., Yiu, M.Y.R. (2010). An AHP approach to assess new product development performance: An exploratory study, International Journal of Management Science and Engineering Management, Vol. 5, No. 3, 210218.

[8] Syamil, A., Doll, W.J., Apigian, C.H. (2004). Process performance in product development: measures and impacts, European Journal of Innovation Management, Vol. 7, No. 3, 205-217, doi: 10.1108/14601060410549892.

[9] Chwastyk, P., Kołosowski, M. (2014). Estimating the cost of the new product in development process, Procedia Engineering, Vol. 69, 351-360, doi: 10.1016/j.proeng.2014.02.243. 
[10] Tarhan, A., Yilmaz., S.G. (2014). Systematic analyses and comparison of development performance and product quality of incremental process and agile process, Information and Software Technology, Vol. 56, No. 5, 477-494, doi: 10.1016/j.infsof.2013.12.002.

[11] Osteras, T., Murthy, D.N.P., Rausand, M. (2006). Product performance and specification in new product development, Journal of Engineering Design, Vol. 17, No. 2, 177-192, doi: 10.1080/09544820500275735.

[12] Bause, K., Radimersky, A., Iwanicki, M., Albers, A. (2014). Feasibility studies in the product development process, Procedia CIRP, Vol. 21, 473-478, doi: 10.1016/j.procir.2014.03.128.

[13] León, H.C.M., Farris, J.A., Letens, G., Hernandez, A. (2013). An analytical management framework for new product development processes featuring uncertain iterations, Journal of Engineering and Technology Management, Vol. 30, No. 1, 45-71, doi: 10.1016/i.jengtecman.2012.11.004.

[14] O’Donnell, F.J., Duffy, A.H.B. (2002). Modelling design development performance, International Journal of Operations \& Production Management, Vol. 22, No. 11, 1198-1221, doi: 10.1108/01443570210450301.

[15] Liker, J.K., Morgan, J. (2011). Lean product development as a system: A case study of body and stamping development at Ford, Engineering Management Journal, Vol. 23, No. 1, 16-28, doi: 10.1080/10429247.2011.11431884.

[16] Rauch, E., Dallasega, P., Matt, D.T. (2015). Axiomatic design based guidelines for the design of a lean product development process, Procedia CIRP, Vol. 34, 112-118, doi: 10.1016/i.procir.2015.07.005.

[17] Pullan, T.T., Bhasi, M., Madhu, G. (2013). Decision support tool for lean product and process development, Production Planning \& Control, Vol. 24, No. 6, 449-464, doi: 10.1080/09537287.2011.633374.

[18] Kirner, K.G.M., Lindemann, U. (2013). Assessing the performance of product development processes in a multiproject environment in SME, In: Chakrabarti, A., Prakash, R. (eds.), ICoRD'13: Lecture Notes in Mechanical Engineering, Springer, India, 797-808, doi: 10.1007/978-81-322-1050-4 63.

[19] Leber, M., Bastič, M., Mavrič, M., Ivanišević, A. (2014). Value analysis as an integral part of new product development, Procedia Engineering, Vol. 69, 90-98, doi: 10.1016/i.proeng.2014.02.207.

[20] Tyagi, S., Choudhary, A., Cai, X., Yang, K. (2015). Value stream mapping to reduce the lead-time of a product development process, International Journal of Production Economics, Vol. 160, 202-212, doi: 10.1016/j.ijpe.2014. $\underline{11.002 .}$.

[21] Agyapong-Kodua, K., Ajaefobi, J.O., Weston, R.H., Ratchev, S. (2012). Development of a multi-product cost and value stream modelling methodology, International Journal of Production Research, Vol. 50, No. 22, 6431-6456, doi: $10.1080 / 00207543.2011 .648777$.

[22] Browning, T.R. (2003). On customer value and improvement in product development processes, Systems Engineering, Vol. 6, No. 1, 49-61, doi: 10.1002/sys.10034.

[23] Chase, J.P. (1999). Value creation in the product development process, Masters thesis in Aeronautics and Astronautics, Massachusetts Institute of Technology, Cambridge, MA, USA.

[24] Browning, T.R. (2000). Value-based product development: Refocusing lean, In: Proceedings of the 2000 IEEE Engineering Management Society, Albuquerque, New Mexico, USA, 168-172, doi: 10.1109/EMS.2000.872495. 


\section{APEM}

\title{
Modeliranje uspešnosti na osnovi analize vrednostni za izboljšanje arhitekture procesa razvoja izdelka
}

\author{
Yin, F.P. ${ }^{a, b}$, Gao, Q. ${ }^{a, b,{ }^{*}}, \mathbf{J i}, X^{a, b}$ \\ ${ }^{a}$ School of Mechanical Engineering, Shandong University, Jinan, China \\ ${ }^{b}$ Key Laboratory of High Efficiency and Clean Mechanical Manufacture, Shandong University, Ministry of Education, Jinan, \\ China
}

\begin{abstract}
POVZETEK
Izboljšanje arhitekture procesa razvoja izdelka (angl. PDP) je učinkovit način za izboljšanje procesa razvoja izdelka. Modeliranje uspešnosti je težavno, saj so kriteriji uspešnosti, kot so stroški in čas razvoja ter kakovost izdelka pogosto izključujoči. Cilj prispevka je uporabiti procesno vrednost kot evalvacijski kazalnik uspešnosti PDP. Obravnavana sta procesna vrednost PDP ter razmerje med procesno funkcijo in ceno procesa, predlagana je kvantitativna metoda. Procesna funkcija je definirana kot učinkovitost procesa, ki upošteva pomembnost vsakega koraka PDP. Za procesno funkcijo sta podani evalvacijski metodi, temelječi na teoriji predelave in uvajanju kakovostne funkcije (angl. QFD). Za prikaz predlaganega modela in za analizo povezave med arhitekturo PDP in procesno vrednostjo PDP je izvedena simulacija, izdelan pa je tudi optimizacijski model za arhitekturo PDP. Z modelom lahko pridobimo primerno arhitekturo PDP za uravnovešenje cene in funkcije izdelka $v$ fazi razvoja izdelka.

\section{PODATKI O ČLANKU}

Ključne besede: Proces razvoja izdelka Uspešnost procesa Arhitektura procesa Analiza vrednosti

Učinkovitost

Modeliranje

*Kontaktna oseba: gaoqi@sdu.edu.cn (Gao, Q.)

Zgodovina članka: Prejet 20. oktobra 2016

Popravljen 15. februarja 2017 Sprejet 18. februarja 2017 